\title{
Analysis, simulation and experimental veri- fication of the throughput of GCRA based UPC functions for CBR streams
}

\author{
F. W. Hoeksema
}

University of Twente, Tele Informatics \& Open Systems Group P.O. Box 217, 7500 AE Enschede, The Netherlands

Tel.: +31 5348927 70,Email: hoeksema@cs.utwente.nl

J. Kroeze

Ericsson Telecommunication

P.B. 8, 5120 AA Rijen, The Netherlands

Tel.: +31 161242 466, Email: etmjohk@etm.ericsson.se

\section{J. Witters}

\section{Alcatel Bell}

Francis Wellensplein 1, B-2018 Antwerp, Belgium

Tel.: +32 324079 27, Email: jwit@rc.bel.alcatel.be

\begin{abstract}
This paper investigates a Generic Cell Rate Algorithm (GCRA) based Usage Parameter Control (UPC) function implementing a discard function for non-conforming cells, thereby establishing a so-called Cell Discard Ratio (CDR) as UPC performance measure. Focusing on Constant Bit Rate (CBR) connections, the UPC transfer characteristics are studied in case of Peak Cell Rate Contract Violations. Also the influence of the Contracted Cell Delay Variation Tolerance value on the CDR performance of the UPC is incorporated in the study.

Algorithmic formulas found by analysis as well as simulation results are compared against the outcome of test-bed measurements. As opposed to the approach in the analysis, the simulation effectively takes into account the delay experienced by the cells accessing the ATM slotted medium, as sole origin of cell delay variation. The applicability of the simulation and the
\end{abstract}


analysis is verified with measurements on real ATM cell streams, using the R2061 EXPLOIT test-bed. Although both the simulation and analysis show clear correspondence with the measurement results, slight deviations are found. These deviations can be partly explained by the slotted nature of ATM networks, which shows the importance of taking this effect into account in the performance analysis of UPC behaviour.

The study results in guide-lines for setting the parameters involved in policing ATM CBR cell streams. These guide-lines are verified by a test-bed measurement with real CBR video data transported over ATM using AAL1.

\section{Keywords}

B-ISDN, ATM, UPC, GCRA, CBR, throughput analysis, simulation, measurements

\section{INTRODUCTION}

The UPC function is an ATM layer traffic control function and is located at the Public UNI (PuUNI) of an ATM network [6]. Its objective is to monitor and control traffic per Virtual Channel Connection or Virtual Path Connection (VCC/VPC) in terms of traffic offered and in terms of validity of the ATM connection. In the sequel the validity of a VCC/VPC is assumed.

Here we focus on policing (popular for "UPC Action") of the Peak Cell Rate (PCR or Rp) of CBR sources. This Peak Cell Rate is defined as the reciprocal of the minimal interarrival time between two consecutive requests to send an ATM_PDU (the 53 byte ATM cell) at the PHY_SAP in ATM Terminal Equipment (TE). The minimal interarrival time is called Peak Emission Interval (PEI or $\mathrm{Tp}$ ), so: $\mathrm{Rp}=1 / \mathrm{Tp}$. The arrival times of the CBR input traffic are given by:

$\left\{\mathrm{ta}_{\mathrm{PHY} \_\mathrm{SAP} @ \mathrm{TE}}[\mathrm{k}]=\mathrm{ta}_{\mathrm{PHY} \_\mathrm{SAP} @ \mathrm{TE}}[1]+(\mathrm{k}-1) \mathrm{Tp} ; \mathrm{k} \geq 1\right\}$

in which ta[k] is the arrival time of the k-th cell of the connection.

As a result of negotiations between TE and network during the connection setup-phase the part of the Traffic Contract necessary for Peak Cell Rate policing is agreed upon. This part consists of a Contracted Peak Cell Rate Rc $(=1 / \mathrm{Tc})$ and a Contracted Cell Delay Variance (CDV) Tolerance $\tau_{c}$. The CDV Tolerance allows for a certain degree of cell clumping, and can be seen as a measure of burstiness of the cell stream at the Pu-UNI. In order to be able to specify unambiguously in the Traffic Contract which cells of a connection are conforming and which cells are not, the Generic Cell Rate Algorithm, as described by the ATM Forum [7], is used as a Conformance Definition. A Conformance Definition can be considered a deterministic means of classifying stochastic source traffic patterns.

The GCRA may not only be used to classify traffic patterns, but also as an algorithm to monitor and control traffic. The UPC function investigated in this article is GCRA(Tc, $\left.\tau_{c}\right)$. Other algorithms which may be used as a UPC function (e.g. moving window, sliding window) have been compared against GCRA in [5]. It is assumed that the UPC function does not buffer more than one cell.

Each cell in the ATM connection is labelled conforming or non-conforming by the UPC function. Cells which are labelled non-conforming are assumed to be discarded in this work (this is not a necessity however, see [6],[7]). 
So, if $\mathrm{X}$ is the number of cells arriving at the ingress of the UPC function since the beginning of the connection, and $\mathrm{Y}$ is the number of (conforming) cells at the egress of UPC function since the beginning of the connection, the number of discarded cells is $\mathrm{X}-\mathrm{Y}$ and we may define a Cell Discard Ratio (CDR) as

$$
\mathrm{CDR}[\mathrm{X}]=\frac{(\mathrm{X}-\mathrm{Y})}{\mathrm{X}} \text {. }
$$

Note that the CDR depends on the number of transmitted cells. The relation with the elapsed time $t$ since the beginning of the connection can be made explicit by defining $X(t)$ and $Y(t)$ and thus

$$
\operatorname{CDR}(\mathrm{t})=\frac{\mathrm{X}(\mathrm{t})-\mathrm{Y}(\mathrm{t})}{\mathrm{X}(\mathrm{t})} \text {. }
$$

As the interest is in the long term behaviour of a UPC function we define

$$
\mathrm{CDR}_{\infty}=\lim _{\mathrm{X} \rightarrow \infty} \mathrm{CDR}[\mathrm{X}]
$$

or

$$
\mathrm{CDR}_{\infty}=\lim _{\mathrm{t} \rightarrow \infty} \operatorname{CDR}(\mathrm{t})
$$

provided that these limits exist.

In the CBR case we have $X(t)=\lfloor t / T p\rfloor+1$ and $\lim _{t \rightarrow \infty} \frac{X(t)}{t}=R p$.

Defining the Passed Cell Rate Ro $=\lim _{t \rightarrow \infty} \frac{Y(t)}{t}$ (provided this limit exists), we find

$$
\mathrm{CDR}_{\infty}=\frac{\mathrm{Rp} \cdot \mathrm{t}-\mathrm{Ro} \cdot \mathrm{t}}{\mathrm{Rp} \cdot \mathrm{t}}=\frac{\mathrm{Rp}-\mathrm{Ro}}{\mathrm{Rp}} \text {. }
$$

If a stream is not conforming to $\operatorname{GCRA}\left(\mathrm{Tc}, \tau_{\mathrm{c}}\right.$ ), e.g. because $\mathrm{Rp}>\mathrm{Rc}$ (or equivalently because $\mathrm{Tp}<\mathrm{Tc}$ ) cells are discarded: the Traffic Contract is violated. The discarding of excess traffic is in accordance with the Traffic Contract and does not contribute to the network Performance degradation allocated to the UPC function [section 3.2.3.2,6].

In this paper the throughput behaviour of a UPC function is considered "ideal" if the amount of discarded cells is proportional to the amount of contract violation (a property called Throughput Fairness (TF) [3],[4]), and if no cells are lost if Tp $\geq$ Tc. So, the "ideal" throughput behaviour is given by

$$
\mathrm{CDR}_{\infty}=1-\frac{\mathrm{Tp}}{\mathrm{Tc}} \text { for } \delta \leq \mathrm{Tp}<\mathrm{Tc} \text { and } \mathrm{CDR}_{\infty}=0 \text { for } \mathrm{Tp} \geq \mathrm{Tc}
$$

Restated otherwise: the "ideal" throughput behaviour of the UPC function is defined as the property to always admit the Contracted PCR, irrespective of the magnitude of the Contract Violation. 
In figure 1 two views of the "ideal" throughput behaviour of a UPC algorithm are depicted.
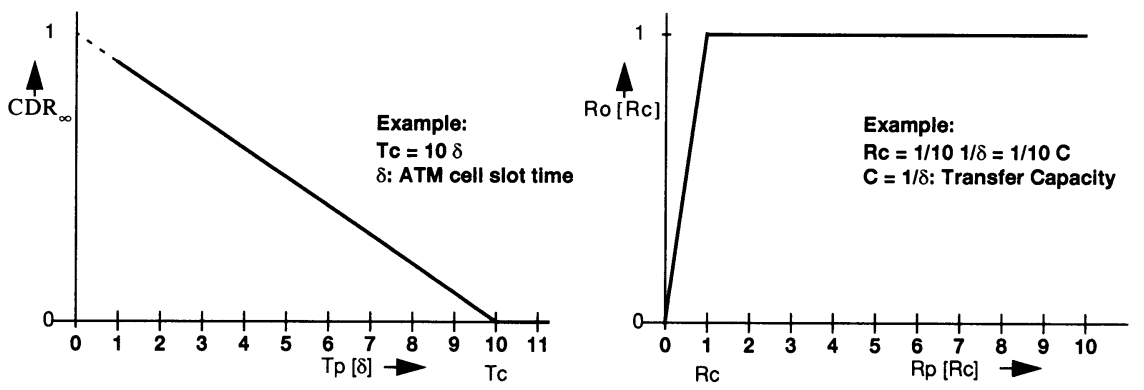

Figure 1. "Ideal" throughput behaviour of a UPC function.

The left side is a graphical representation of formula (4), while the right side of the figure shows the normalised Passed Cell Rate as a function of the Peak Cell Rate (both normalized with respect to the Contracted Cell Rate). The figure can be easily derived rewriting formulas (3) and (4) as

$$
\frac{\mathrm{Ro}}{\mathrm{Rc}}=\left(1-\mathrm{CDR}_{\infty}\right) \cdot \frac{\mathrm{Rp}}{\mathrm{Rc}} \text {. }
$$

$\delta$ denotes the ATM Cell Slot Time, the inverse of the Cell Transfer Capacity C [cell/s] of the link at the PHY_SAP (e.g. C $=155.5210^{6} /(53.8)$ cell/s for STM-1). It is assumed that $\mathrm{Rp} \leq \mathrm{C}$, or equivalently $\mathrm{Tp} \geq \delta$.

Note that our UPC function acts instantaneously on a per-cell basis, there is no shaping included (which might be beneficial from the user's point of view, provided that introduction of delay is acceptable for the service).

In this article we compare the throughput behaviour of the GCRA based UPC function with respect to Throughput Fairness, using results from analysis, simulation and test-bed measurements. Guidelines for the selection of the Contracted CDV Tolerance will be presented.

In the next section the terminal configuration, the source traffic and causes of CDV are presented. In section 3 the results of analysis and guidelines for the selection of the Contracted CDV Tolerance are given. The simulation results are presented in section 4 and test-bed measurements in section 5 . In section 6 the guidelines are verified by policing a CBR video stream.

In section 7 results of analysis, simulation and test-bed measurement are compared. Section 8 contains our conclusions. 


\section{TERMINAL CONFIGURATION, SOURCE TRAFFIC AND CDV}

A CBR source may be connected to ATM Terminal Equipment (TE) via AAL1 at a VCC/VPC endpoint. This source produces a stream of requests to send an ATM_SDU (ATM cell payload, 48 byte; interaction primitive ATM_SDU_Data.request). It is assumed that the previously mentioned stream (the user data component [section 2.3.3,6] of the tagged connection) is multiplexed at the ATM layer in the TE with Operations, Administration and Maintenance (OAM) cells and cell streams of other connections. After multiplexing, the cells of the tagged connection are considered to be shaped by a shaper, resulting in a stream with PEI Tp.

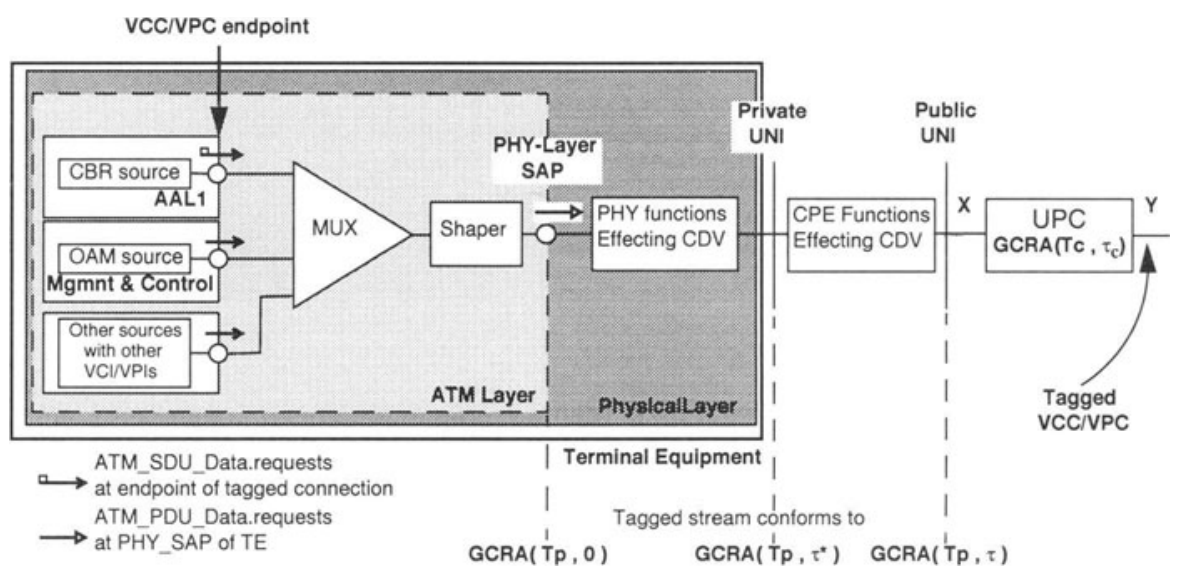

Figure 2. Terminal Example.

In figure 2 a terminal example is given, using an adaptation of both figure 4 of I.371 [6] and the PCR Reference model of the ATMF UNI 3.0 specification [7].

At the PHY_SAP of TE the cell stream (stream of ATM_PDU_Data.requests) of the tagged connection has an PEI of $\mathrm{Tp}$, a result of the shaper. However, this is not the case any more at the Public UNI (stream of ATM_PDU_Data.indications at the PHY_SAP of Pu-UNI). Cell Delay Variation is introduced by the following mechanisms:

- 1/. Due to the ATM multiplexing with OAM cells and cells of other connections at the PHY_SAP in TE some cells may be delayed.

- 2/. The ATM_PDU_Data.indications at the PHY_SAP of the Pr-UNI only occur at discrete time instances. This so called slotted nature of ATM networks causes CDV.

- 3/. Due to insertion of PHY layer overhead the ATM cell slot times may not be of equal length.

- 4/. Between the Pr-UNI and Pu-UNI CDV may be introduced by Customer Premises Equipment (CPE). 
Note that the precise nature of CDV causes in the CPE is left unspecified here. It may consist of, but not be limited to: ATM multiplexing, the slotted nature of ATM networks or PHY layer overhead.

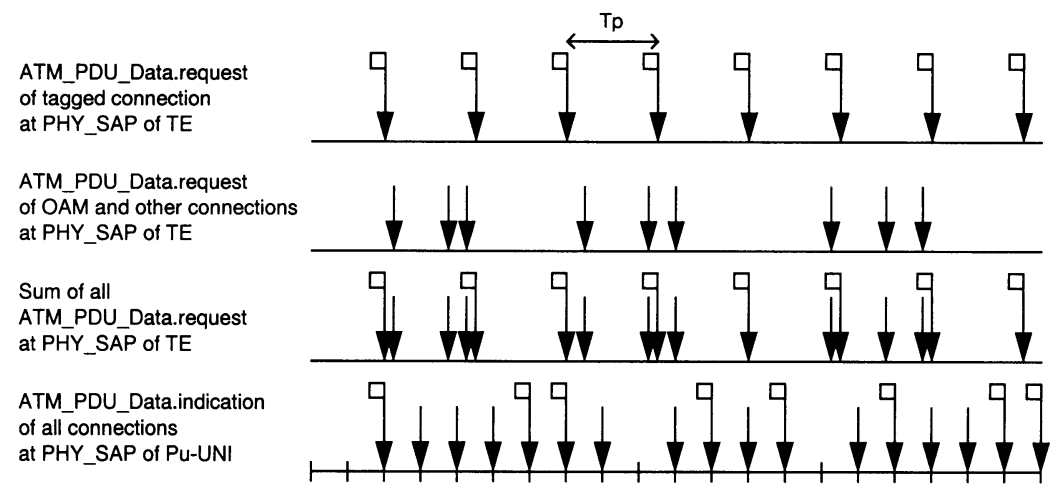

Cells of the tagged connection only:

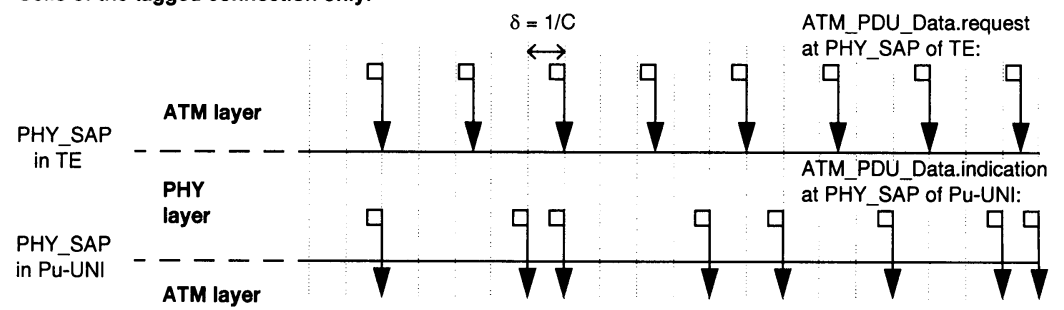

Figure 3. CDV of cells of the tagged connection (no CPE caused CDV).

In Figure 3 an example of the introduction of CDV is given (CDV cause 1/. to 3/., no CDV caused by 4/.). Note that the shaper guarantees a PEI of Tp at the PHY_SAP of TE for the tagged connection. Competing ATM_PDU_Data.requests at the PHY_SAP in TE are assumed to be in continuous time (see the summation in the figure above).

In the following analysis (section 3) all these four causes of CDV are neglected. In the simulation (section 4) however, the slottedness (cause 2/.) is taken into account. During the measurements with an ATM traffic generator CDV causes 3/. and 4/. may be present (section 5). Finally, the traffic from a TV Terminal Adapter may experience all CDV causes mentioned above (see section 6). 


\section{ANALYSIS}

In figure 4 the Cell Streams of figure 2 are given, only showing CDV caused by the slottedness of ATM networks (CDV cause 2/.)

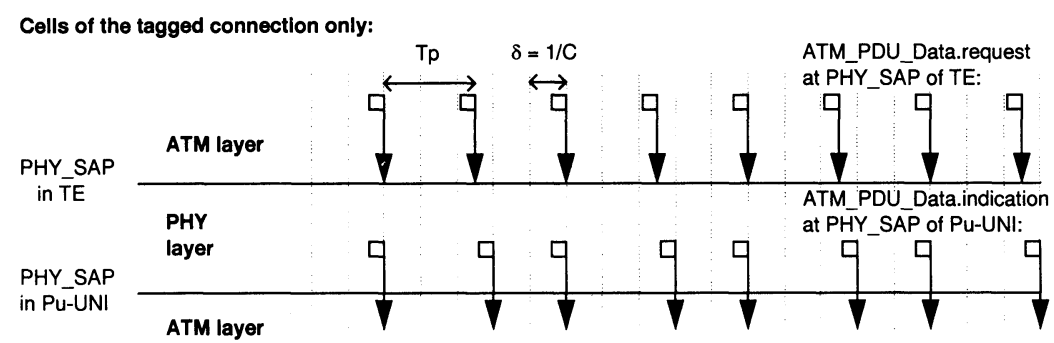

Figure 4. CDV caused by the slotted nature of ATM only.

The slotted nature of the ATM networks is neglected in the presented expressions but is important in comparing our results with results of simulation experiments [3],[4] and test-bed measurements [12]. The assumption we make is that (apart from a constant delay):

$\mathrm{ta}_{\mathrm{PHY} \_\mathrm{SAP} @ \mathrm{Pu} \text {-UNI }}[\mathrm{k}]=\mathrm{ta}_{\mathrm{PHY} \_S A P @ T E}[\mathrm{k}]$

while, when $2 /$. is taken into account (see figure 4):

$\mathrm{ta}_{\mathrm{PHY} \_ \text {SAP@Pu-UNI }}[\mathrm{k}]=\left\lceil\mathrm{ta}_{\mathrm{PHY} \_ \text {SAP@TE }}[\mathrm{k}] / \delta\right\rceil \delta$

Only the situation of Traffic Violation is investigated, thus $\delta<\mathrm{Tp}<\mathrm{Tc}$, no cells are lost if $\mathrm{Tp} \geq \mathrm{Tc}$. In the sequel we will present $\mathrm{CDR}_{\infty}$ as a function of $\mathrm{Tc}, \mathrm{Tp}$ and $\tau_{\mathrm{c}}$; a derivation of these results is given in [13].

Three cases can be distinguished, depending on the Contracted CDV $\tau_{\mathrm{c}}$ :

- $\tau_{\mathrm{c}}=0$

In this case:

$\mathrm{CDR}_{\infty}=1-1 / \mathrm{N}$, with $\mathrm{N}=\lceil\mathrm{Tc} / \mathrm{Tp}\rceil$

- $0<\tau_{\mathrm{c}}<\mathrm{Tp}$

In this case an algorithmic solution was found in [13]. It consists of finding P and M (both required to be integer and as small as possible) which satisfy:

$\mathrm{M} \mathrm{Tc} / \mathrm{Tp}+1 \leq \mathrm{P}<\mathrm{M} \mathrm{Tc} / \mathrm{Tp}+1+\left(1-\tau_{\mathrm{c}} / \mathrm{Tp}\right)$

or equivalently: 
$\mathrm{P}<\left(\mathrm{M} \mathrm{Tc}-\tau_{\mathrm{c}}\right) / \mathrm{Tp}+2 \leq \mathrm{P}+\left(1-\tau_{\mathrm{c}} / \mathrm{Tp}\right)$

As $\mathrm{P}>\mathrm{M}$ the algorithm should start with $\mathrm{M}=1$.

Then:

$\mathrm{CDR}_{\infty}=1-\mathrm{M} /(\mathrm{P}-1)$

- $\tau_{c} \geq T p$

It can be shown that the throughput behaviour is "ideal" in this case, so:

$$
\mathrm{CDR}_{\infty}=1-\frac{\mathrm{Tp}}{\mathrm{Tc}}
$$

Now, we will present some results of the analysis which can be directly compared to the results of simulation (section 4) and test-bed measurements (section 5). Comparisons will be deferred until the simulation and test-bed results are presented.

In figure 5 the CDR as a function of the PEI Tp is presented for Tc $=10 \delta$ for different values of the Contracted CDV Tolerance $\tau_{\mathrm{c}}$. The "ideal" throughput curve is shown too (see figure 1).
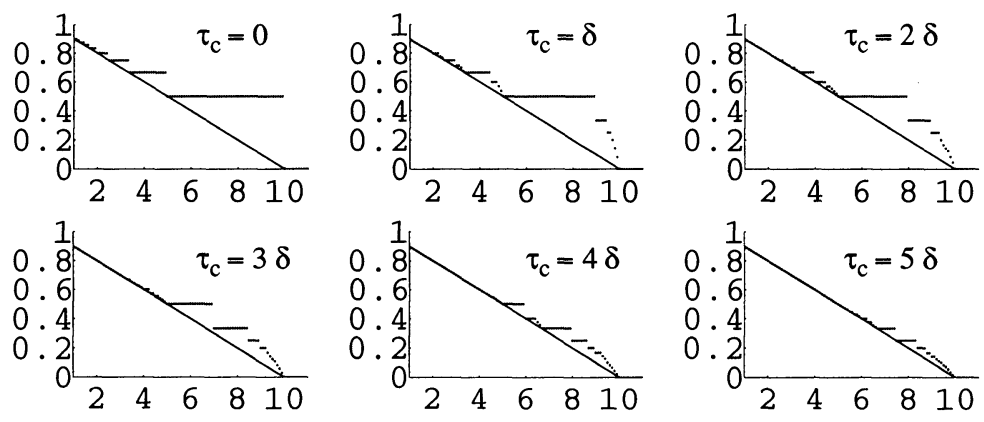

Figure 5. $\mathrm{CDR}_{\infty}$ as a function of $\mathrm{Tp}[\delta]$ for $\mathrm{Tc}=10 \delta$. Different values of $\tau_{\mathrm{c}}$. 
Figure 6 shows the Passed Cell Rate Ro as a function of the Peak Cell Rate Rp for both the "ideal" behaviour and the behaviour for $\tau_{\mathrm{c}}=8 \delta$ with Tc $=63.75 \delta$.

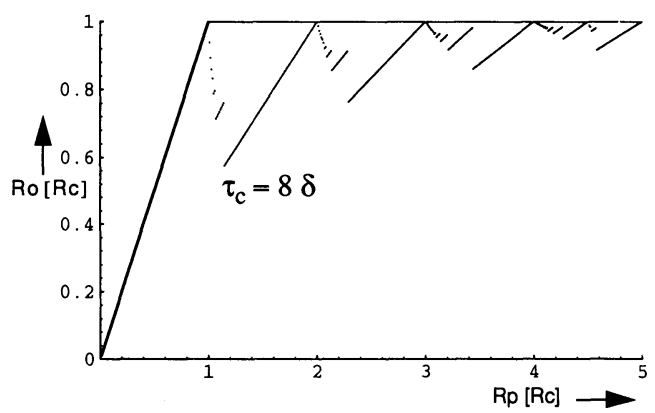

Figure 6. Passed Cell Rate Ro as a function of PCR.

Figure 7 offers a closer look at the previous figure, and shows the influence of a change in Contracted CDV Tolerance.

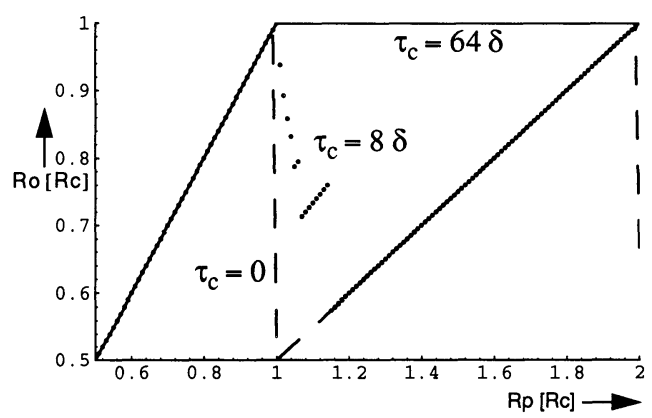

Figure 7. Passed Cell Rate Ro as a function of PCR.

From our analysis it is clear that the throughput behaviour for $\tau_{\mathrm{c}}=0$ is far from "ideal". Increasing $\tau_{\mathrm{c}}$ in the region $0<\tau_{\mathrm{c}}<\mathrm{Tp}$ improves the throughput behaviour of the GCRA based UPC function, but does not realize "ideal" throughput behaviour either. Only when $\tau_{c} \geq \mathrm{Tp}$ the situation is reached in which the Throughput Fairness property holds.

These observations allow us to provide guidelines for the selection of the Contracted CDV Tolerance if Throughput Fairness is required:

In the connection set-up phase, the user presents his requested PEI $\mathrm{Tu}=\mathrm{Tp}$ and requested CDV Tolerance $\tau_{u}=0$ to the network. For the UPC function to perform "ideal" throughput behaviour our analysis shows that the contracted values should be $\mathrm{Tc}=\mathrm{Tp}$ and $\tau_{\mathrm{c}}=\mathrm{Tp}=\mathrm{Tc}$. If a user intents to violate the Traffic Contract and specifies the required PEI as $\mathrm{Tu}=\mathrm{Tp}$ ' $>\mathrm{Tp}$ (the actual PEI) and required CDV Tolerance as $\tau_{\mathrm{u}}=0$, the network will select Tc $=\mathrm{Tp}$ ' and 
$\tau_{\mathrm{c}}=\mathrm{Tp}$ ' $=\mathrm{Tc}$ if "ideal" UPC throughput behaviour is required. From our analysis we notice that the Contracted CDV Tolerance is too high in this case, it could be $\tau_{\mathrm{c}}=\mathrm{Tp}<\mathrm{Tp}$ '. As the network has no idea of the intentions of the user the only "fair" selection of $\tau_{\mathrm{c}}=\mathrm{Tc}=\mathrm{Tp}$ '.

In [3] it is shown that the following approximation of the CDR holds when $\Delta$ is fairly small and $\mathrm{Tc} \geq \tau_{\mathrm{c}}$ :

$$
\mathrm{CDR}_{\infty} \cong \mathrm{Tc} * \Delta / \tau_{\mathrm{c}} \quad, \text { where } \Delta=(\mathrm{Tc}-\mathrm{Tp}) / \mathrm{Tc}
$$

a result which is in accordance with [1], [4] and [2], where a more general relation is given,

$$
\mathrm{CDR}_{\infty}=1-\mathrm{d} /(\mathrm{d}+1), \text { with } \mathrm{d}=\left\lceil\tau_{\mathrm{c}} /(\mathrm{Tc}-\mathrm{Tp})\right\rceil
$$

which is in agreement with the algorithm in (8), (9) and (10). However, note that (14) only holds for $0<\tau_{c}<\mathrm{Tp}$ and $\mathrm{Tc} / 2 \leq \mathrm{Tp}<\mathrm{Tc}$ as is stated as a (strong) conjecture in [13]. Using (14) it can be shown that the approximation in (13) is in fact an upper bound to $\mathrm{CDR}_{\infty}$, which becomes tighter as $\Delta \ll<1$. So, for small contract violations (13) shows that for $\tau_{c} \geq \mathrm{Tc}$ indeed $\mathrm{CDR}_{\infty} \cong \Delta$.

\section{SIMULATION}

The GCRA based UPC function is analysed by simulation techniques taking into account the delay experienced by cells accessing the ATM slotted medium (CDV cause $2 /$., section 2). ATM cells are generated periodically by a CBR source (on the real time axis). Due to the transfer to the ATM slotted medium, cells can be delayed while others are not influenced, thus introducing a limited CDV (see figure 4). This cell flow is then passed to the UPC function where the GCRA is executed and the CDR is measured.

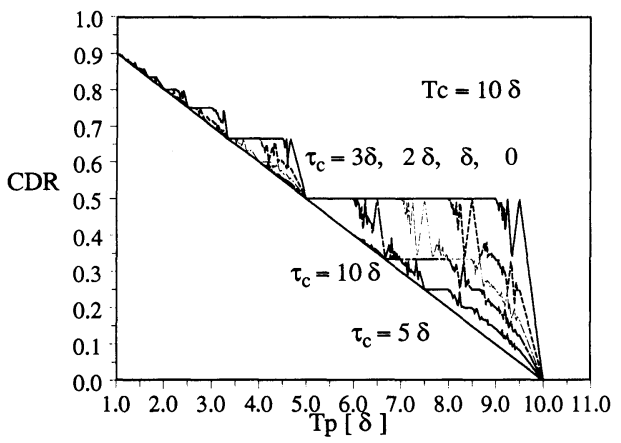

Figure 8. CDR as a function of Tp. Tc $=10 \delta$.

The CDR in case of a Contracted PEI Tc $=10 \delta$ is shown in figure 8 as a function of the actual PEI and of the Contracted CDV tolerance $\tau_{\mathrm{c}}$. Comparing the results with the analytical ones (see figure 5), there are a lot of similarities. The major differences are the oscillations marking the transitions between two regions of constant CDR which occur at PEIs which are not an integer 
multiple of the cell slot time $\delta$. In that case some cells experience a delay due to the access to the ATM slotted medium while others are not delayed, so the arrivals at the UPC function are not strictly periodical any more (e.g. figure 4 , lower part)

Indeed, a CBR connection which exceeds the Contracted PCR Rc by only a small amount immediately experiences a severe Cell Discard Ratio, disproportionate with the degree of misbehaviour $\Delta$ (see (13)). On the other hand a source which sends twice the amount of allowed traffic sees a CDR of $50 \%$, a value in accordance with the TF property.

From the simulations it became clear that a GCRA based UPC function tuned with the Contracted PEI Tc and $\tau_{c}=\lceil\mathrm{Tc} / \delta\rceil \delta \geq \mathrm{Tc}$ obtains Throughput Fairness. Similar findings have been reported in [8] when observing the UPC responsiveness characteristics.

\section{MEASUREMENTS}

The initial aim of the experiments was to validate the correct operation of the implemented UPC. function, see [12]. Here the measurement results are used to verify the applicability of the analysis and simulations in the previous sections, using a real ATM network.

The experiments were performed at the EXPLOIT test-bed, which has four ATM switches, several terminals with appropriate adapters and measurement equipment (see [10]). The Police Function Board (PFB) can implement several UPC mechanisms, like Leaky Bucket (LB), jumping window, moving window etc. Here we use the LB mechanism as UPC function. The PFB is part of one module in the Remote Unit (RU) which is one of the available switches at the test-bed.

Figure 9 shows a simplified view of the experiment configuration. As indicated by the arrows,

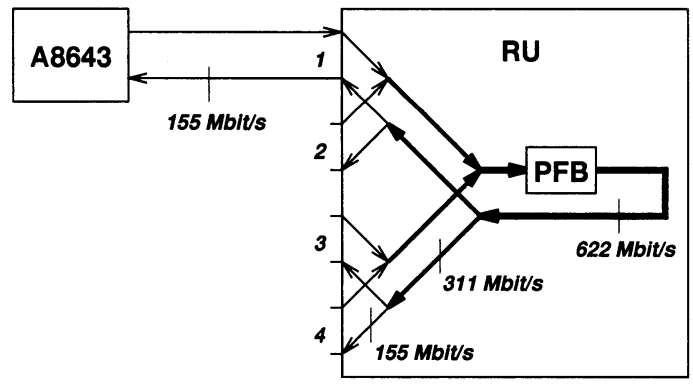

Figure 9. EXPLOIT Experimental configuration.

RU: Remote Unit (a switch); PFB: Police Function Board.

the traffic from four input ports is multiplexed to one stream entering the PFB. Cells may therefore always experience delay variations within the RU before being policed. Even with only one source connected, CDV is caused by Operations Administration and Maintenance (OAM) traffic inside the RU (interpreting the part of the RU before the PFB as CPE allows us to model this as CDV cause 4/.). Traffic leaving the PFB is routed back through the RU and demultiplexed to one of the four output ports of this module. 
The ATM traffic streams were generated, received and analysed with the Alcatel 8643, a PC-card with memory for 8192 assigned ATM cells. This memory can be played out repetitively, while counters keep track of the number of sent and received cells.

The implemented LB is a discrete state realisation of the GCRA and is defined by three discrete parameters: splash, leak rate and bucket limit. The bucket level is also discrete and ranges from 0 to 65535 units. With every passed cell, a splash (from 0 to 255 units) is added to the bucket level, which leaks with a constant rate, selectable by factors of two from $2^{-14}$ to

$2^{7}$ units/slot. A cell arriving at the PFB is discarded if the bucket level exceeds the bucket limit, which can be set from 0 to 65504 in steps of 32 . These calculations are all performed by the Police Criterion Chip (PCC) [11].

The PFB parameters are related to the GCRA parameters by:

$\mathrm{Tc}=$ splash $/$ leak rate $\quad \tau_{\mathrm{c}}=$ bucket limit $/$ leak rate

With appropriate parameters, the PCC can act as a cell counter such that the number of received and discarded cells at the PFB are known. This allows to verify that all generated cells make it to the PFB and that cells are only lost due to discards and not by e.g. buffer overflow. Instead of the PFB parameters we will in the following use the parameters Tc and $\tau_{c}$, both expressed in slots at $155.52 \mathrm{Mbit} / \mathrm{s}$.

Some initial measurements where the PCR Rp and Contracted PCR Rc were exactly the same $(\mathrm{Tp}=\mathrm{Tc}=64 \delta)$ revealed a Cell Discard Ratio of approximately $2.10^{-6}$, although $\tau_{\mathrm{c}}$ was chosen large enough to allow all possible CDV. These discards are due to frequency deviations between the crystal of the free-running clock of the A8643 and the clock crystal of the RU, to which the PFB is synchronised. Note that, although this causes cell discards, it is not one of the CDV causes mentioned in section 2 . To prevent these discards, a slightly lower value of $\mathrm{Tc}=63.75 \delta$ is used for further measurements.

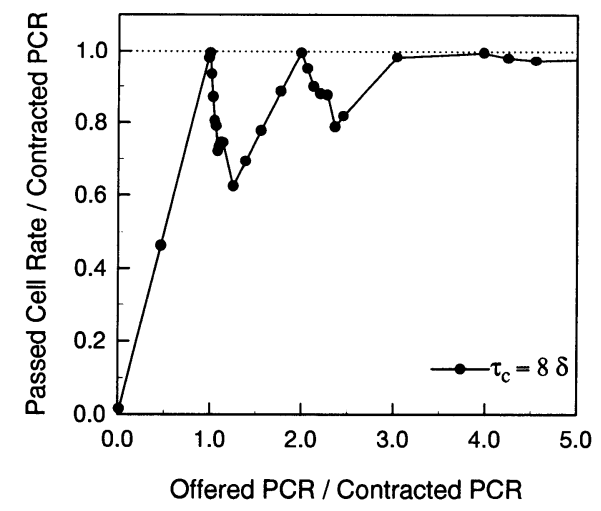

Figure 10. Passed Cell Rate Ro as a function of PCR. 
Figure 10 depicts the measured throughput of the LB as a function of the PEI Tp of the A8643 for a Contracted PEI Tc of 63.75 slots (at $155.52 \mathrm{Mbit} / \mathrm{s}$ ). The Contracted CDV Tolerance is $\tau_{c}=8 \delta$. Mind that the dots in figures 10 and 11 are the only measurement points, the line pieces just connect the dots in the correct order. The generated cell streams of the A8643 only have Tp values which are integer multiples of the cell slot time, so that the cell inter-departure times at the egress of A8643 are strictly constant, e.g. the pattern as shown in the upper part of figure 4.

However, on the way to the RU and the PFB, the cell stream probably suffers CDV by multiplexing with OAM cells in the RU (CDV cause 4\% in section 2). ATM multiplexing in TE (cause 1/.) does not play a role. Due to the integer values of Tp the slottedness of ATM networks (cause $2 /$.) is not taken into account. Physical layer overhead (cause 3/.) may play a role.

The throughput is defined as the ratio of the actual cell rate of the A8643 and the Contracted PCR, multiplied by the ratio of the numbers of passed and sent cells (so Ro/Rc, see (5)). Both axes are normalised to the Contracted PCR. This figure can be directly compared to figure 6.

It is clear from figure 10 that the throughput depends both on the offered PCR and on the CDV. tolerance. As long as the actual PCR does not exceed the Contracted PCR, all cells pass the LB. If however the actual PCR increases above the contracted value, the passed traffic is limited to the Contracted PCR, even if the A8643 generates at full link rate. Two measurements with a PCR slightly below and above the contracted one can not be distinguished in the plot, but the results are as expected. We see again that the punishments imposed by the LB are not proportional to the violation. This is shown more clearly in figure 11, which covers the region of small contract violations (compare with figure 7). The dips in the throughput curve vanish when the CDV tolerance is increased to the Contracted PEI.

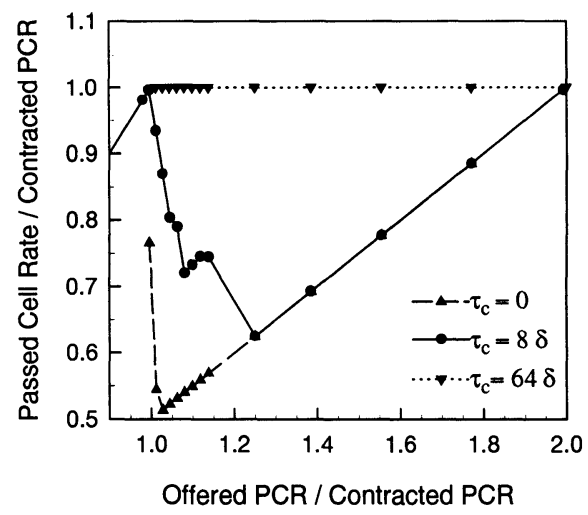

Figure 11. Passed Cell Rate Ro as a function of PCR. 


\section{PEAK CELL RATE CONTROL FOR DIGITAL VIDEO}

The experience gained in policing CBR sources was used to police one of the real sources available at the EXPLOIT testbed, namely the TV signal (audio and video signal) which originates from the TV-Terminal Adapter (TV-TA). The (composite) TV signal is converted to a digital signal with a bit rate of $34.368 \mathrm{Mbit} / \mathrm{s}$ for the video signal and $0.96 \mathrm{Mbit} / \mathrm{s}$ for the audio signal. These "reference" signals will eventually be packetized into ATM cells according to the AAL1 adaptation layer standard for CBR traffic. Before that however, Forward Error Correction (FEC) combined with bit or byte interleaving is used for error correction [9].

For the video component a Reed-Solomon code is used which allows for an error correction of at most 4 cells out of 64 consecutive cells. So the source characteristics undergo changes because of the introduction of FEC overhead and (in AAL1) signal timing recovery. This results into a ATM physical rate of $41.366 \mathrm{Mbit} / \mathrm{s}$ for the video signal and of $1.49 \mathrm{Mbit} / \mathrm{s}$ for the audio component.

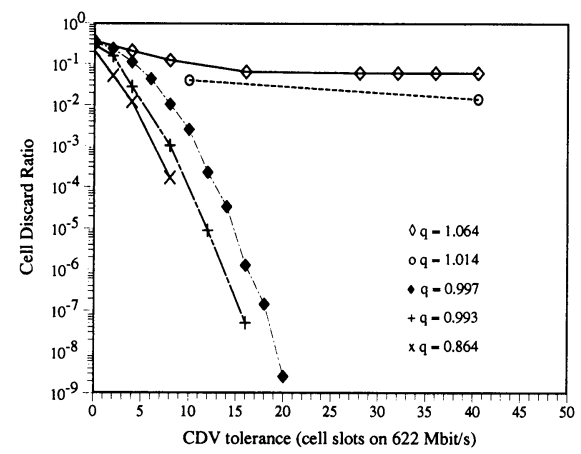

Figure 12. Measured CDR as a function of Contracted CDV Tolerance. $q=R p / R c$

In the policing experiments, the TV-set is continuously sending traffic which is passed through the RU (where the PFB is located) to a TV screen. The Contracted PCR (the PCR which is used for policing the source) and the Contracted CDV tolerance are changed for each experiment and the Cell Discard at the PFB is measured. In figure 12 the CDR is plotted as a function of the Contracted CDV tolerance $\tau_{c}$ and for different PCRs of the video source which are expressed in terms of the Contracted PCR Rc $(q=R p / R c, R p=41.366 \mathrm{Mb} / \mathrm{s})$. The Contracted PEIs Tc can be derived from the q-values by:

$\mathrm{Tc}=\mathrm{q} \mathrm{Tp}=\mathrm{q} \mathrm{C} / \mathrm{Rp} \delta$.

This results in Tc $=16.0 \delta, 15.2 \delta, 15.0 \delta, 14.9 \delta$ and $13.0 \delta$ for the q-values in figure 12.

If the actual PCR of the source is higher than the Contracted PCR (the theoretically Contract Violation region, defined by $\mathrm{q}>1$ ), then changing the $\mathrm{CDV}$ tolerance (within reasonable limits) has only a very small influence on the CDR (this can be seen in the upper right part of figure 12). Once the CDV tolerance is larger than the inverse of the Contracted PCR $(=\mathrm{Tc})$, the UPC 
discards exactly the excess amount of traffic (this result was found also earlier, see figure 11, in which the passed cell rate Ro equals the Contracted PCR Rc, provided $\tau_{c}$ is large enough).

This observation leads to a method which allows us to derive experimentally the actual PCR of a policed CBR source, once the CDR is measured. In case of a Contract Violation and a CDV Tolerance value larger than the Contracted PCR, we observed that $R o=R c$, so using (5) we find:

$\mathrm{Rp}=\mathrm{Rc} \cdot \frac{1}{1-\mathrm{CDR}}$

Indeed, if this method is applied to the experimental results, we find a PCR of

41.3666 Mbit/s on the $155.52 \mathrm{Mbit} / \mathrm{s}$ link and so a "reference" signal rate of $34.368 \mathrm{Mbit} / \mathrm{s}$ exactly as mentioned earlier. The same procedure was successfully used to determine the PCR of the audio component of the TV signal.

If the actual PCR of the source is below the Contracted PCR (the theoretically no Contract. Violation region, defined by $q<1$, the lower left part of figure 12), all cells should be accepted if the assumptions used for the analysis hold. From these measurement results we conclude that at least one of the mentioned CDV causes (section 2) is present. The clock mismatch problem (section 5) is believed not to influence these results, since the clocks of the TV-TA and PFB were synchronised.

Since apparently, in realistic situations, cells always experience some CDV, increasing the CDV tolerance seems necessary. It drastically reduces the CDR, even if no discards are expected (being in the theoretically no Contract Violation region, $\mathrm{q}<1$ ). This is especially true if the PCR approaches the Contracted PCR $(\mathrm{q} \cong 1)$. In order to draw further conclusions we need to model the RU more precisely.

\section{COMPARISON OF ANALYSIS, SIMULATION AND MEASUREMENTS}

When comparing the results of analysis and simulations, the impact of the slottedness of ATM networks on the throughput behaviour of a GCRA based UPC function becomes clear. Mind that the CDR values at integer values of $T p$ (see figure 8) should equal the values of $\mathrm{CDR}_{\infty}$ at integer values of $\mathrm{Tp}$ in figure 5, something which graphically appears to be the case (and shows that the number of simulated arrivals was large enough).

Comparing the results of the test-bed measurements with those of the analysis shows a good agreement between the two (at least graphically). From this we conclude that the effects on discards caused by the clock-mismatch between A8643 and PFB are effectively undone by a slight adaptation of the Contracted PEI Tc. Note that the impact of the slottedness of ATM was not encountered in these experiments, as the cell arrival process had only PEIs at a multiple of the cell slot time. Apparently, the OAM traffic generated by the RU (CDV cause 4/., section 2) does not alter the measured throughput function significantly.

The CBR video experiment (section 6) showed that increasing the Contracted CDV Tolerance, even in the theoretically no Contract Violation case is beneficial from a UPC discard point of view. This was not found using the other approaches, which shows the importance of 
the measurements. However, the necessity of detailed modelling of the system on which the measurements were performed is clear.

\section{CONCLUSIONS}

In this paper the throughput behaviour of a GCRA based UPC function for ATM traffic control has been studied by analysis, simulation and measurements.

The analysis resulted in an algorithm to compute the Cell Discard Ratio $\mathrm{CDR}_{\infty}$ for infinite length CBR traffic streams. The CDR is a function of the contracted GCRA parameters Tc, $\tau_{c}$ and the PEI Tp of the cell stream. The analysis does not take any CDV generating mechanism into account, and assumes that cells arrive in continuous time, spaced Tp apart, at the ingress of the UPC function.

The results of the simulation are in-line with the analysis results as far as comparable. The simulation takes the CDV due to the slottedness of the physical layer into account. The influence of the slottedness on the throughput behaviour of the UPC function is limited but significant. No results of analysis have been found by the authors which take the slottedness of ATM into account, although the methods presented in [13] may prove valuable in solving this issue.

Measurements with an ATM traffic generator and an implemented UPC function show that the analysis and simulation apply to real ATM networks. Analysis and simulation show remarkable resemblance with the measurement results. Effects of clock-mismatch can be undone effectively by a slight adaptation of Tc, at least in the case where no CDV due to slottedness is present.

Measurements with real ATM audio and video traffic however, show that apart from the slottedness of ATM networks, other CDV causes have their influence on the throughput of the UPC function as well. This is especially apparent if the PCR approaches the Contracted PCR. In this case increasing $\tau_{\mathrm{c}}$ drastically reduces the cell discard. In order to explain these observations, the experimental configuration has to be modelled in greater detail.

Guidelines for the selection of $\mathrm{Tc}$ and $\tau_{\mathrm{c}}$ were presented, based on the results from analysis and simulation. These guidelines proved useful when applied to a realistic situation, although also in the theoretically no Contract Violation case, $\tau_{c}$ has to be increased. As to how much exactly, guidelines still need to be found. This is an item for further study.

The research may have a follow-up by extending analysis and simulation by inclusion of more CDV causes. The two approaches should be tested with measurements in systems that are described at a level of detail required by the models used. 
A study consisting of analysis, simulation and measurements of the policing mechanism for traffic using other ATM Transfer Capabilities (a.k.a. ATM service categories or connection types) seems necessary, e.g. for Variable Bit Rate traffic, for Available Bit Rate traffic or for Signalling traffic.

Policing of the Sustainable Cell Rate is another candidate subject for further study.

\section{ACKNOWLEDGEMENTS}

The measurements have been carried out as part of the RACE project R2061 EXPLOIT. The authors would like to thank all the people who contributed to EXPLOIT, especially work package 3.1 who supplied the measurement results as presented in this paper and T. Renger for providing the plots.

\section{REFERENCES}

[1] S. Liu, T. Chen, V.K. Samalam and J. Ormond. "Performance Analysis of GCRA for CBR Sources". ATM Forum contribution 94-0182, March 1994.

[2] B.G. Kim and I.G. Niemegeers. "Assessment of Traffic Control Schemes in ATM Networks". Memoranda Informatica 94-46, TIOS 94-16. ISSN 0924-3755. August 291994.

[3] J.Witters, S.Muti and G.H. Petit. "Performance Analysis of a VSA-based UPC Function for Constant Bit Rate Sources". Alcatel-Bell Internal Research Report, TTD_068/JW_930330, Ed. 1, 30/03/93.

[4] R. Wilts, J. Witters and G.H. Petit. "Throughput Analysis of a UPC Function Monitoring Misbehaving CBR Sources". Proc. Second Workshop on Performance Modelling and Evaluation of ATM Networks. 4-7 July, 1994. Bradford, UK. pp. 39/1-39/17.

[5] H. Hemmer. "Evaluation of UPC Functions for Peak Cell Rate Enforcement". European Transactions on Telecommunications, Vol. 5, nr.2. March - April 1994. pp. 27-31.

[6] ITU-T Recommendation I.371: Traffic Control and Congestion Control in B-ISDN, Frozen Issue - Paris, March 1995.

[7] ATM forum UNI specification version 3.0. Prentice Hall, 1993.

[8] J. Witters, G.H. Petit \& S. Muti. "Responsiveness Characteristics of a Usage Parameter Control Function based on the Virtual Scheduling Algorithm Monitoring Misbehaving Constant Bit Rate Sources". ITC Seminar on Digital Communication and Network Management, St. Petersburg, Russia, June 15-20, 1993.

[9] H.Hessenmuller, S.Nunes. "A terminal Adapter for High-Quality Audio and Video Signals to be used in a B-ISDN based on ATD". Proc. of the International Symposium on Broadcasting Technology, Beijing, September 1991.

[10] M. Potts. "EXPLOITation of an ATM Testbed for Broadband Experiments and Applications". Electronics \& Communication Engineering Journal, December 1992, pp. 385-393.

[11] K. van der Wal, M. Dirksen, D. Brandt. "Implementation of a Police Criterion Calculator based on the Leaky Bucket Algorithm". Proc. of the IEEE Globecom '93, Houston, Nov. 29 - Dec. 2, 1993, pp. 713- 718. 
[12] A. Bohn Nielsen, R. Elvang, H. Hemmer, H. Pettersen, J. Kroeze, T. Renger, J. Witters. "Results of experiments on traffic control using test equipment". RACE project R2061 EXPLOIT, Deliverable 18, 30.06.94.

[13] F.W. Hoeksema. "On the performance of GCRA based UPC functions for Peak Cell Rate policing". Proc. B-ISDN Teletraffic Modelling Symposium, Antwerp, Alcatel Bell, February $17,1995$.

\section{BIOGRAPHY}

Fokke Hoeksema studied electrical engineering at the University of Twente. He received his MSc degree in 1987 after which he joined Philips Research. He participated in the design of a high-quality high-resolution still-image coding system and contributed to modelling of CCD image sensors.

In 1989 he joined the department of electrical engineering of the University of Twente, where he worked in the field of subband image-coding and packet video (BOAT project, together with KPN research).

Currently, as an assistant professor in the Centre of Telematics and Information Technology (CTIT), he is working on design and analysis of architectures and protocols for Broadband Telecommunication networks. He investigated ATM traffic control mechanisms and is currently working on QoS improvement methods that use the outcomes of performance measurements to close control loops in ATM based networks.

John Kroeze (born 1965) studied electrical engineering at the University of Twente, which resulted in a MSc degree in 1992, after working on his thesis at the Dutch PTT Research laboratory in Leidschendam.

He worked for three years at the Computing Science Institute of the University of Nijmegen, participating in the European RACE II project R2061 EXPLOIT, for which he was involved in ATM traffic control experiments.

Recently he has joined Ericsson Telecommunications in Rijen, the Netherlands, where he works at the Intelligent Networks Application Laboratory.

Johan Witters studied physics at the University of Antwerp (Belgium) where he obtained his Ph.D in 1992 in the area of Quantum Physics with a theoretical study of superfluid helium.

In the same year he joined the research centre of Alcatel Bell in Antwerp.

Since then, he is working on ATM traffic control. His main interest is on Usage Parameter Control both from a theoretical as well as an experimental point of view.

The theoretical aspects involve both simulations and queuing techniques. For the experimental side, he has participated in RACE project EXPLOIT.

Now he is active in the ACTS project EXPERT. Currently he works on policing for the Available Bit Rate Service. 